ARTICLE

\title{
Structure of the far-red light utilizing photosystem I of Acaryochloris marina
}

Tasuku Hamaguchi (1) ${ }^{1,9}$, Keisuke Kawakami ${ }^{2,8,9 凶}$, Kyoko Shinzawa-Itoh ${ }^{3,9}$, Natsuko Inoue-Kashino (i) ${ }^{3}$, Shigeru Itoh (1) ${ }^{4}$, Kentaro Ifuku (10 ${ }^{5}$, Eiki Yamashita (1) ${ }^{6}$, Kou Maeda ${ }^{3}$, Koji Yonekura (1) ${ }^{1,7 凶} \&$ Yasuhiro Kashino (iD ${ }^{3 凶}$

Acaryochloris marina is one of the cyanobacterial species that can use far-red light to drive photochemical reactions for oxygenic photosynthesis. Here, we report the structure of $A$. marina photosystem I (PSI) reaction center, determined by cryo-electron microscopy at 2.58 $\AA$ resolution. The structure reveals an arrangement of electron carriers and light-harvesting pigments distinct from other type I reaction centers. The paired chlorophyll, or special pair (also referred to as P740 in this case), is a dimer of chlorophyll $d$ and its epimer chlorophyll $d^{\prime}$. The primary electron acceptor is pheophytin $a$, a metal-less chlorin. We show the architecture of this PSI reaction center is composed of 11 subunits and we identify key components that help explain how the low energy yield from far-red light is efficiently utilized for driving oxygenic photosynthesis.

\footnotetext{
${ }^{1}$ Biostructural Mechanism Laboratory, RIKEN SPring-8 Center, Sayo, Hyogo, Japan. ${ }^{2}$ Research Center for Artificial Photosynthesis (ReCAP), Osaka City University, Sumiyoshi-ku, Osaka, Japan. ${ }^{3}$ Graduate School of Life Science, University of Hyogo, Ako-gun, Hyogo, Japan. ${ }^{4}$ Department of Physics, Graduate School of Science, Nagoya University, Nagoya, Japan. ${ }^{5}$ Division of Integrated Life Science, Graduate School of Biostudies, Kyoto University, Sakyo-ku, Kyoto, Japan. ${ }^{6}$ Laboratory of Supramolecular Crystallography, Institute for Protein Research, Osaka University, Suita, Osaka, Japan. ${ }^{7}$ Institute of Multidisciplinary Research for Advanced Materials, Tohoku University, Aoba-ku, Sendai, Japan. ${ }^{8}$ Present address: Biostructural Mechanism Laboratory, RIKEN SPring-8 Center, Sayo, Hyogo, Japan. ${ }^{9}$ These authors contributed equally: Tasuku Hamaguchi, Keisuke Kawakami, Kyoko Shinzawa-Itoh. ${ }^{凶}$ email: kawakami.k@spring8.or.jp; yone@spring8.or.jp; kashino@sci.u-hyogo.ac.jp
} 
$\mathrm{P}$ hotosynthesis is driven by two types of photoreaction systems, namely type I and type II reaction centers. Photosystems I (ref. ${ }^{1}$ ) and II (ref. ${ }^{2}$; PSI and PSII, belonging to type I and type II reaction centers, respectively) work sequentially in oxygenic photosynthesis in plants and cyanobacteria ${ }^{3}$. They typically use chlorophyll (Chl) $a$ (Supplementary Fig. 1) as the major pigment that absorbs visible light $(670-700 \mathrm{~nm}$ in the redlight region; Supplementary Fig. 2). Anoxygenic photosynthetic bacteria use bacteriochlorophylls (BChl) that absorb 800-900-nm light in addition to blue/ultraviolet light and have either a type I or type II reaction center. Acaryochloris marina (A. marina) was found as a cyanobacterial species that uses Chl $d$ (Supplementary Fig. 1), which absorbs 700-750-nm light in vivo ${ }^{4}$ (Supplementary Fig. 2). Later, several cyanobacteria were found that induce a limited amount of $\mathrm{Chl} f$ for capturing far-red light under the farred light condition ${ }^{5-7}$ (Supplementary Fig. 2, and see below). A. marina was isolated from colonial ascidians, which harbor mainly Chl $a$-type cyanobacteria, resulting in an environment with low visible light and high far-red light. Analogous to the cortex effect within a lichen body $^{8}$, high-energy blue light absorbed by the Chls likely has diminished intensity within the symbiotic host ascidians. A. marina has exploited this environment with the niche-filling introduction of Chl $d$ that absorbs far-red light ${ }^{9,10}$. The discovery of $A$. marina prompted intensive research into the mechanism of this low-energy-driven system. Although the organism also contains $\mathrm{Chl} a$ at $\sim 5 \%$ relative abundance, spectroscopic and pigment analyses revealed that it is Chl $d$ that plays a central role in the photoreaction ${ }^{4,9,11}$. With the acquisition of Chl $d$, A. marina harnesses far-red light, the energy of which is lower than that of visible light that is utilized in most oxygenic photosynthetic reactions. Chl $d$ has a peak wavelength of absorption at $\sim 697 \mathrm{~nm}$ in methanol (Supplementary Fig. 3; socalled Qy band; 700-750 nm in vivo, Supplementary Fig. 2), which is longer than the $665.2 \mathrm{~nm}$ of Chl $a$ (670-700 nm in vivo, Supplementary Figs. 2 and 3 ) by $\sim 30 \mathrm{~nm}$. This means that the photon energy absorbed is $\sim 80 \mathrm{mV}(10 \%)$ lower. How PSI and PSII of A. marina drive the similar photochemical reactions that occur in the Chl $a$-dependent systems of other oxygenic photosynthetic organisms has been a long-standing puzzle.

PSI generates reducing power for NADPH production by accepting electrons originating from $\mathrm{PSII}^{3}$. PSI of A. marina has a similar subunit composition to that of the PSIs of other oxygenic photosynthetic organisms $^{12,13}$ (Supplementary Table 1). However, the peak wavelength of the light-induced redox difference absorption spectrum of the paired Chls, the so-called special pair, in A. marina PSI is longer $(740 \mathrm{~nm})$ than that of Chl $a(700 \mathrm{~nm})$ in the PSI of plants and typical cyanobacteria ${ }^{14}$ (Fig. 1a). The special pair P740 have been assumed to be a heterodimer of Chl $d$ and $d^{\prime}$ (refs. ${ }^{15,16}$ ) as in P700, which is composed of a heterodimer of Chl $a / \mathrm{Chl} a^{\prime}$ in all other PSIs (Fig. 1a) ${ }^{1,17}$. Cofactors of the PSI electron transfer chain in A. marina (Fig. 1b) and the reduction potential of P740 are also similar to those of P700 in other organisms $^{12,13,18}$.

The far-red light-absorbing $\mathrm{Chl}$, Chl $f$, is also utilized in some cyanobacteria to capture far-red light (Supplementary Fig. 2). How $\mathrm{Chl} f$, as well as $\mathrm{Chl} d$, has succeeded in expanding harvesting the low-energy far-red light region is of great interest ${ }^{5-7}$. Chl $f$ is only induced under far-red light conditions, and even then makes up less than $\sim 10 \%$ of total Chls in PSI (seven Chl $f$ and $83 \mathrm{Chl} a$ in Halomicronema hongdechloris $\mathrm{PSI}^{19}$ ), and the locations of these Chl $f$ molecules is still debated ${ }^{20,21}$. Recent cryo-EM analyses show that Chl $f$ is not part of the chargeseparating pigments, including and following the special pair $^{19,22,23}$. Thus, Chl $f$ in these PSIs is likely entirely responsible for light harvesting. High-resolution ${ }^{24}$ and ultrafast ${ }^{25}$ spectroscopic studies support this. Still, PSII in some cyanobacteria may use Chl $f$ (and/or Chl $d$ ) for electron transfer when grown under far-red light conditions ${ }^{21,26}$. In contrast to Chl $f$-carrying PSIs $19,20,22,23,25,27$, Chl $d$ in $A$. marina PSI is always induced even under natural white light and does, in fact, take the place of the special pair Chls in the photochemical reaction chain. The Chl $d$ driven photoreaction occurs by low-energy far-red light corresponding to the Qy band of Chl $d$. Chl $d$ also absorbs high-energy blue light, corresponding to the Soret band, but the resulting excited state relaxes to a lower excited state corresponding to the Qy level for the photochemical reaction. In this sense, the Chl $d$ driven PSI-system is unique among photochemical reaction centers, utilizing as it does far-red light level energy.

To date, several structures of type I reaction centers have been determined from higher plants 28,29 , green algae ${ }^{30,31}$, red algae ${ }^{32}$, diatoms $^{33}$, cyanobacteria $1,19,22,23,34,35$, and an anoxygenic bacterium $^{36}$. All structures of type I reaction centers including Chl $f$-carrying cyanobacteria ${ }^{19,22,23}$ have Chl $a$ in the electron transfer chain with the one exception, namely, the one from the anoxygenic bacterium that has $\mathrm{BChl} g^{\prime}$ and $8^{1}-\mathrm{OH}-\mathrm{Chl} a$ (ref. ${ }^{36}$ ). The PSI of $A$. marina with $\mathrm{Chl} d$ in its electron transfer chain for utilizing low-energy light directly for the photochemistry is thus unique.

Here, we show the structure of the PSI trimer isolated from $A$. marina revealed by cryo-electron microscopy (cryo-EM) at 2.58 $\AA$ resolution. The special pair P740 is a dimer of Chl $d$ and its epimer Chl $d^{\prime}$, and the primary electron acceptor is pheophytin (Pheo) $a$ not Chl $a$ nor Chl $d$, which is embedded in membrane protein complex composed of 11 subunits. The structure helps gain insight into the mechanism for the utilization of far-red light (Fig. 1b).

\section{Results and discussion}

Overall structure, protein subunits, and cofactors of $A$. marina PSI. The PSI trimer complex was isolated from A. marina. Detailed methods of sample preparation, data processing, and structural refinement by cryo-EM, and biochemical data, are presented in Supplementary Information (Supplementary Figs. 412 and Supplementary Tables 1-4). The model of the PSI trimer was refined to give a correlation coefficient and Q-score, which are indices for validating the correctness of a model, of 0.80 and 0.65 , respectively (Supplementary Table 4 ). These index values can be considered reasonable at $2.58 \AA$ resolution (Supplementary Fig. 11).

The PSI trimer has dimensions of $100 \AA$ depth, $200 \AA$ length, and $200 \AA$ width, including the surrounding detergent micelle (Fig. 2). The overall structure resembles those of PSI trimers reported for other cyanobacteria $1,22,23,35$. The root-mean-square deviations using secondary structure matching between the monomer model (chains A-M) and Protein Data Bank (PDB) structures 1JB0 of Thermosynechococcus elongatus ${ }^{1}$, $5 \mathrm{OY} 0$ of Synechocystis sp. PCC $6803^{35}$, and 6KMX of H. hongdechloris ${ }^{19}$ are $1.09 \AA, 1.05 \AA$, and $1.11 \AA$, respectively.

The A. marina PSI monomer contains 11 subunits (PsaA, PsaB, PsaC, PsaD, PsaE, PsaF, PsaJ, PsaK, PsaL, PsaM, and Psa27), all of which could be assigned to the cryo-EM density map (Fig. 2, Supplementary Figs. 6 and 13, and Supplementary Table 1). PsaX (a peripheral subunit in other cyanobacteria such as T. elongatus), $\mathrm{PsaG}$, and $\mathrm{PsaH}$ (subunits in higher plants) were missing in the density map, consistent with the absence of their genes from the $A$. marina genome ${ }^{10}$. The name Psa27 was given to a subunit protein in $A$. marina that has low sequence identity (29.4\%) with PsaI of $T$. elongatus (Supplementary Table 5) ${ }^{12}$. Here, we found that Psa27 is in the same location as PsaI, a transmembrane alpha helix, in $T$. elongatus. Psa27 also contributes to the structural stabilization of the PSI trimer just 


\section{a Cyanobacteria and Higher plants}

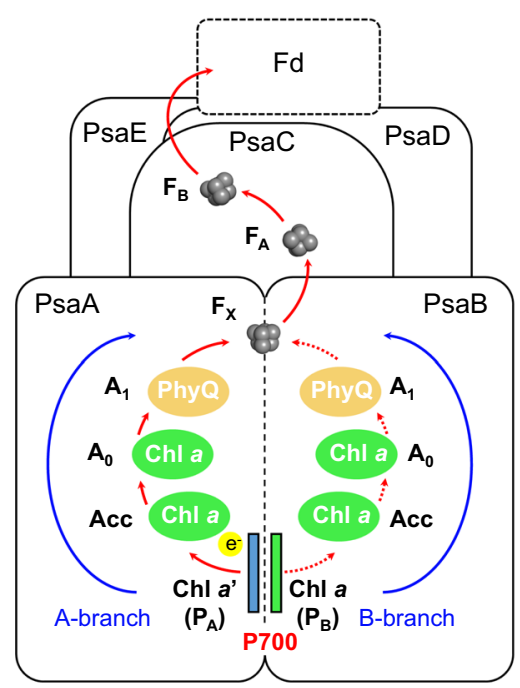

b

\section{Acaryochloris marina}

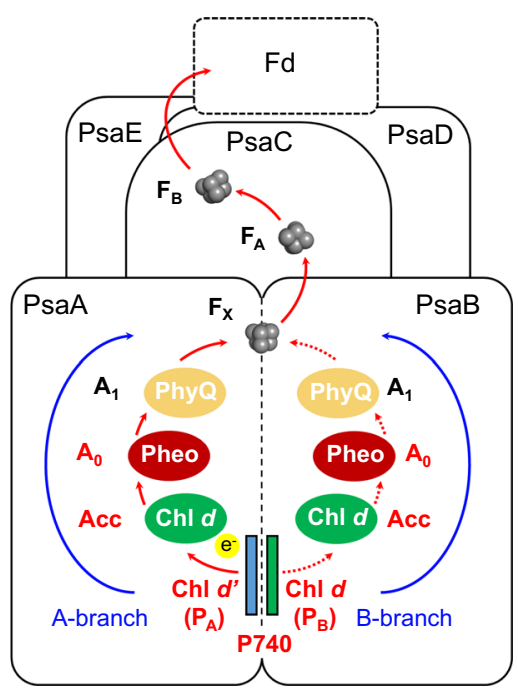

Fig. 1 Comparison of electron transfer chains in PSI. a Cyanobacterial and higher plant PSI. b A. marina PSI identified in this work. In cyanobacterial and higher plant PSI, electrons released from the paired Chls, so-called special pair, of P700, a heterodimer of $\mathrm{Chl} a$ and $a^{\prime}$, are transferred to ferredoxin (Fd) to reduce $\mathrm{NADP}^{+}$via $\mathrm{Acc}(\mathrm{Chl} a), \mathrm{A}_{0}(\mathrm{Chl} a), \mathrm{A}_{1}(\mathrm{PhyQ}), \mathrm{F}_{\mathrm{X}}$ (iron-sulfur center), and $\mathrm{F}_{\mathrm{A}} / \mathrm{F}_{\mathrm{B}}$ (iron-sulfur center). The components are arranged in a pseudo- $\mathrm{C}_{2}$ axis on a heterodimer reaction center protein complex (PsaA/PsaB) and the two routes (A- and B-branch located on PsaA and PsaB, respectively) are thought to be equivalent. Acc accessory chlorophyll, $A_{0}$ primary electron acceptor, $A_{1}$ secondary electron acceptor, $F_{X}, F_{A}$, and $F_{B}$ iron-sulfur centers, Phy $Q$ phylloquinone. In A. marina PSI, the electron transfer chain is the same, but some cofactors differ from those in the PSIs of other organisms; the Chls of special pair P740 are $\mathrm{Chl} d / d^{\prime}$, while Acc could be $\mathrm{Chl} d$ (see text for detail) and $\mathrm{A}_{0}$ is pheophytin (Pheo) $a$.

as PsaI does in T. elongatus. Thus, we concluded that Psa27 and PsaI are in effect the same subunit.

The loop regions of $\mathrm{PsaB}$ (residues 290-320 and 465-510) were not identified due to disorder because of the absence of PsaX, suggesting that PsaX stabilizes these regions in other cyanobacteria. Most of subunits PsaE, PsaF, and PsaK were modeled using polyalanine because of poor regional map quality (Supplementary Figs. 13 and 14, and Supplementary Table 1), possibly suggesting that some part of those subunits dissociated during sample preparation.

The cofactors assigned (Supplementary Fig. 15) are $70 \mathrm{Chl} d$ (Supplementary Data 1), 1 Chl $d^{\prime}$ (an epimer of Chl $d$ ), 12 acarotenes ( $\alpha$-Car) but no $\beta$-Car (Supplementary Table 6) ${ }^{10,14}, 2$ Pheo (a derivative of Chl; however, unlike Chl, no $\mathrm{Mg}^{2+}$ ion is coordinated by the tetrapyrrole ring), 2 phylloquinones (PhyQs), 3 iron-sulfur clusters, 2 phosphatidylglycerols (PGs), 1 monogalactosyl diacylglycerol (MGDG), and 84 water molecules. Two molecules of Pheo were assigned as Pheo $a$ on the basis of pigment analysis (Supplementary Table 6). In addition, a small amount of Chl a (approximately one Chl a per Chl $d^{\prime}$ ) was detected by pigment analysis (Supplementary Table 6). In this study, we assigned the Chls of A. marina PSI as Chl $d$. This is because the amount of Chl $a$ in the A. marina PSI is minimal, and it is not possible to distinguish between Chl $d$ and Chl $a$ at $2.58 \AA$ resolution, as is described later.

In the A. marina PSI, a large number of Chl $d$ and $a$-Car harvest light energy and finally transfer the energy to P740. The arrangements of Chl $d$ and $\alpha$-Car in A. marina resemble those of Chl $a$ and $\beta$-Car, respectively, in T. elongatus, as shown in Supplementary Fig. 16. The pigments are numbered according to the nomenclature of $\mathrm{Chl} a$ in T. elongatus ${ }^{37}$ (Supplementary Data 1). However, there are differences in some amino acid residues surrounding the cofactors, slight gaps in the arrangement of the cofactors, and absence of some Chls in the A. marina PSI compared with those of T. elongatus (Supplementary Table 5 and Supplementary Figs. 16-18). For example, Phe49/J in A. marina, the counterpart of His39/J that is an axial ligand of Chl $a 88$ in T. elongatus PSI, explains the absence of the corresponding Chl $d$ (capitalized letters following the slash (/) indicate the subunit names, such as PsaA or PsaB; Supplementary Fig. 16d). Owing to the absence of PsaX in A. marina, the Chl $d$ corresponding to Chl a 95 (refs. ${ }^{1,38}$ ) in T. elongatus whose axial ligand is Asn23/X is also missing. The absence of Chl $a 94$ is seen in some other reported PSI structures, such as Synechocystis sp. PCC 6803 (ref. ${ }^{35}$ ). The arrangements of pigments adjacent to Psa27 (PsaI) and PsaL, whose sequence identity with other cyanobacterial PsaLs is low, are specific to A. marina (Supplementary Fig. 16a, black dashed line region, and Supplementary Figs. 17 and 18). Chl $d 38,52,53$, and the ring ( $\alpha$ or $\varepsilon$ ) of Car4007 near Chl $d 53$ are set within the surrounding structure differently from those in $T$. elongatus (Supplementary Fig. 17a). These differences when compared with the structure of $T$. elongatus help explain the specific features of light harvesting in A. marina. In addition, the C3-formyl groups of some Chl $d$ in the A. marina PSI form hydrogen bonds with their surrounding amino acid residues (Supplementary Data 1 and Supplementary Fig. 18). These characteristic structural features will be important in future theoretical studies of the light-harvesting mechanism in $A$. marina.

Although the amount of Chl $d$ per Chl $d^{\prime}$ determined by pigment analysis $(67.0 \pm 0.66, n=5$ of independently prepared PSI) and assigned by structural analysis (70 including one or two Chl $a)$ is lower in this study than those in previous studies $(145 \pm$ 8 , ref. ${ }^{14}$ and $97.0 \pm 11.0$, ref. $\left.{ }^{12}\right)$, the semi-stoichiometric amount of Phe $a$ at $1.92 \pm 0.022(0.3 \pm 0.2$ per reaction center in a previous study $\left.{ }^{12}\right)$ is consistent with our structural analysis. Notwithstanding the fact that the local resolution of some parts of the outer region is somewhat lower, the PSI was stable enough to keep its 
a

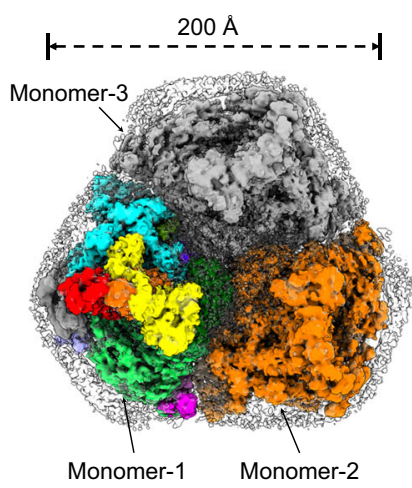

b

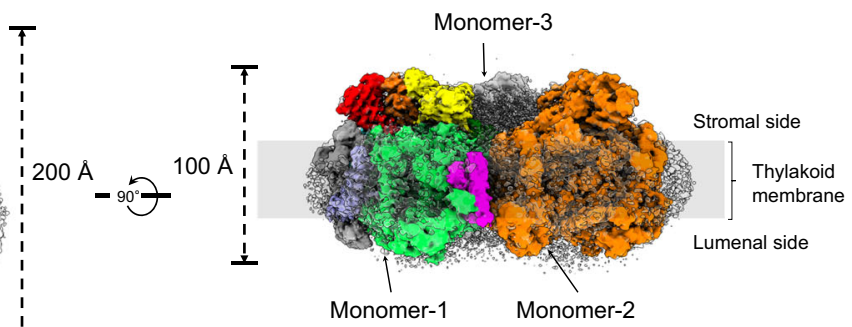

d

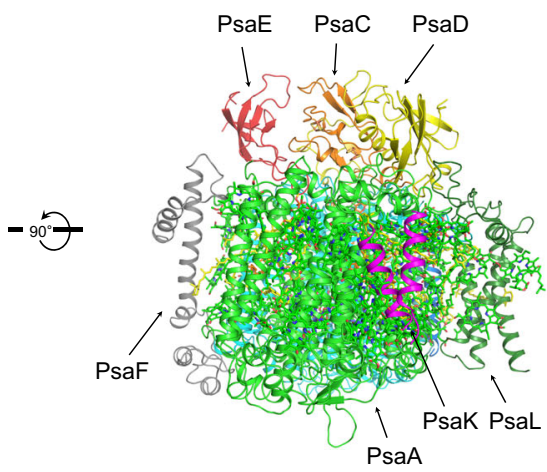

Fig. 2 Overall structure of the photosystem I (PSI) trimer from Acaryochloris marina. a Cryo-electron microscopy (cryo-EM) density map of PSI trimer viewed from the stromal side perpendicular to the membrane plane. Monomer-1, multicolored; monomer-2, orange; monomer-3, gray; detergent micelle, cloudy gray. $\mathbf{b}$ Cryo-EM density map of PSI trimer viewed from the side of the membrane plane. $\mathbf{c}$ Structural model of PSI monomer with view in the same direction as in a. PsaA, green; PsaB, cyan; PsaC, orange; PsaD, yellow; PsaE, red; PsaF, gray; PsaJ, light blue; PsaK, magenta; PsaL, dark green; PsaM, teal blue; Psa27, blue. $\mathbf{d}$ Structural model of PSI monomer with view in the same direction as in $\mathbf{b}$.

integrity for 5 days (Supplementary Fig. 5). Chls that were unable to be assigned in A. marina PSI compared with those in $T$. elongatus PSI are shown by marking N/D in the column of the ID number in Supplementary Data 1. Such Chls can be recognized by the chlorins in transparent gray in Supplementary Fig. 16. Chls corresponding to Chl-88 and Chl-94 in T. elongatus appear to be absent in A. marina PSI. The numbers of Chls in the PSI of Chl $f$ carrying Fischerella thermalis (89, ref. ${ }^{22}$ ) and H. hongdechloris (90, ref. ${ }^{19}$ ) are also lower than those of T. elongatus PSI (96, ref. ${ }^{1}$ ). The type I reaction center of Heliobacterium modesticaldum carries a much smaller amount of Chl species; 60 molecules per reaction center ${ }^{36}$.

Due to the somewhat lower resolutions of the outer regions of the A. marina PSI, we mainly focus on the structure and function of the central part of $A$. marina PSI at high local resolution, that is, P740 and the electron transfer components.

Electron transfer components. The configuration of the electron transfer chain in A. marina PSI is similar to that of the PSI from T. elongatus (Fig. 1), although cofactor compositions are different. The important assigned cofactors involved in electron transfer (Fig. 1b) are four Chls, two Pheos, two PhyQs $\left(\mathrm{A}_{1}\right)$, and three iron-sulfur clusters $\left(\mathrm{F}_{\mathrm{X}}, \mathrm{F}_{\mathrm{A}}\right.$, and $\mathrm{F}_{\mathrm{B}}$; Fig. 3a). The Chls and PhyQs are arranged in two branches, the $\mathrm{A}$-branch and the B-branch, which are related by a pseudo- $\mathrm{C}_{2}$ axis as in other type I reaction centers, and are stabilized by amino acid residues of subunits PsaA and PsaB. The Chls of special pair P740 are Chl $d^{\prime}\left(\mathrm{P}_{\mathrm{A}}\right)$ and Chl $d\left(\mathrm{P}_{\mathrm{B}}\right)$, which are coordinated by residues His678/A and His657/B, respectively (Fig. 3b, c). The distance between the ring planes (the $\pi-\pi$ interaction distance) of Chl $d^{\prime}$ and Chl $d$ is $3.5 \AA$
(Fig. 3d; $3.6 \AA$ in T. elongatus). Two Tyr residues, Tyr601/A and Tyr733/A, are positioned within hydrogen bonding distance of $\mathrm{P}_{\mathrm{A}}$ as observed in PSI from T. elongatus.

In A. marina PSI, we identified three water molecules (W1 W3) around $\mathrm{P}_{\mathrm{A}}$. They form hydrogen bonds with surrounding amino acid residues (Tyr601/A, Ser605/A, Asn608/A, Ser741/A, and Try745/A) and one Chl $d$ (Chl $d$ 32; Fig. 3b, c). For the T. elongatus $\mathrm{P}_{\mathrm{A}}$, only one water molecule forms hydrogen bonds with surrounding amino acid residues (Tyr603/A, Ser607/A, Ile610/A, Thr743/A, and Phe747/A) and Chl $a^{\prime}\left(\mathrm{P}_{\mathrm{A}}\right)$. There are no water molecules around $\mathrm{P}_{\mathrm{B}}$ in A. marina, but some water molecules surround $\mathrm{P}_{\mathrm{B}}$ without forming hydrogen bonds. The arrangement of these water molecules differs from that of $T$. elongatus PSI, and this difference around $\mathrm{P}_{\mathrm{B}}$ may come from the difference in two amino acid residues: Val594/B in A. marina vs. Thr597/B in T. elongatus, and Asn598/B in A. marina vs. His601/ $\mathrm{B}$ in $T$. elongatus. The hydrogen bonding pattern around $\mathrm{P}_{\mathrm{A}}$ probably contributes to the charge distribution ratio $\left(\mathrm{P}_{\mathrm{A}} \cdot+1\right.$ $\left.\mathrm{P}_{\mathrm{B}} \cdot{ }^{+}\right)^{1,39}$, and therefore, is likely different in the two organisms. The midpoint potential values, $E_{\mathrm{m}}\left(\mathrm{P}_{\mathrm{A}}\right)$ and $E_{\mathrm{m}}\left(\mathrm{P}_{\mathrm{B}}\right)$, are influenced by the protein environment, in particular by the presence of charged residues. The $E_{\mathrm{m}}$ difference, $E_{\mathrm{m}}\left(\mathrm{P}_{\mathrm{A}}\right)-E_{\mathrm{m}}$ $\left(\mathrm{P}_{\mathrm{B}}\right)\left(=\Delta E_{\mathrm{m}}\right)$, is an important factor in determining the $\mathrm{P}_{\mathrm{A}}{ }^{+}+$/ $\mathrm{P}_{\mathrm{B}} \cdot+$ ratio $^{40}$.

The orientations of the formyl group in $\mathrm{P}_{\mathrm{A}}\left(\mathrm{Chl} d^{\prime}\right)$ and $\mathrm{P}_{\mathrm{B}}$ (Chl $d$ ) were identified by considering the distribution of the cryo-EM density (Fig. 4a, b). No amino acid residues and pigments capable of forming hydrogen bonds with these formyl groups were found in the vicinity of $\mathrm{P}_{\mathrm{A}}$ and $\mathrm{P}_{\mathrm{B}}$. This suggests that these formyl groups form hydrogen bonds with the $\mathrm{C} 5 \mathrm{H}$ atom in 
a

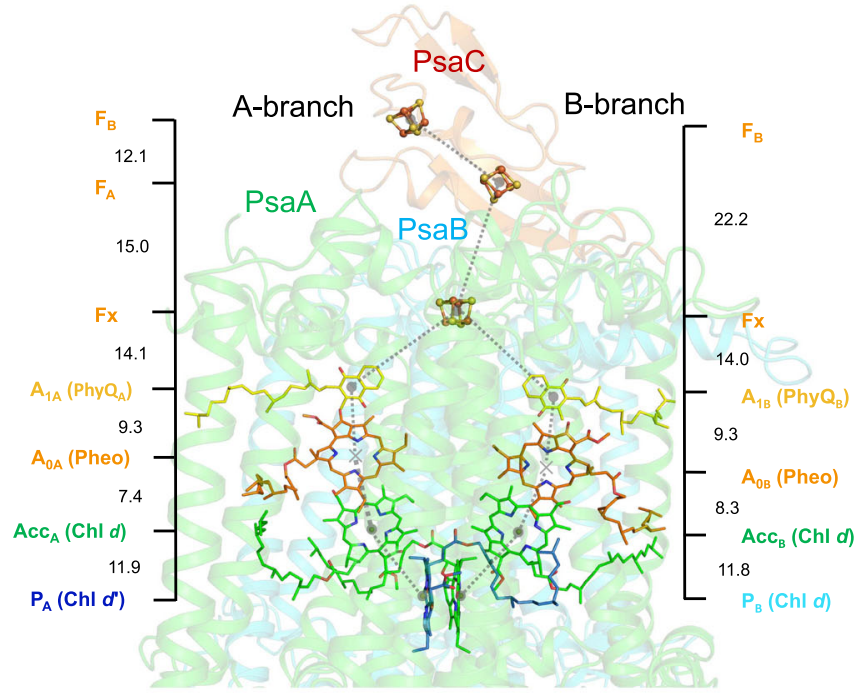

b

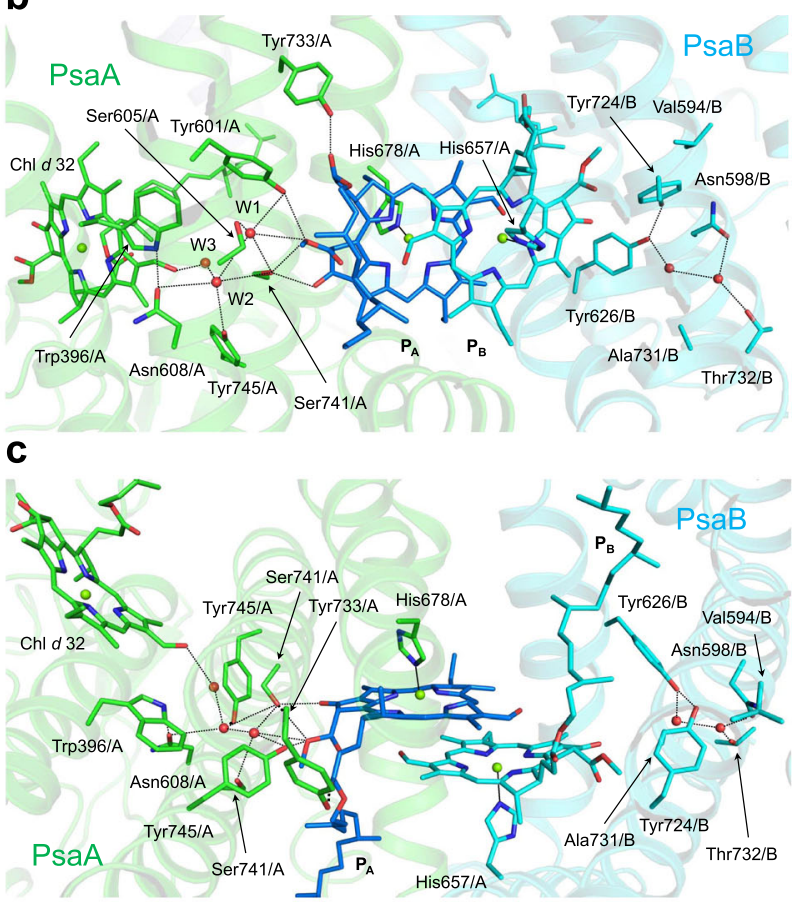

d

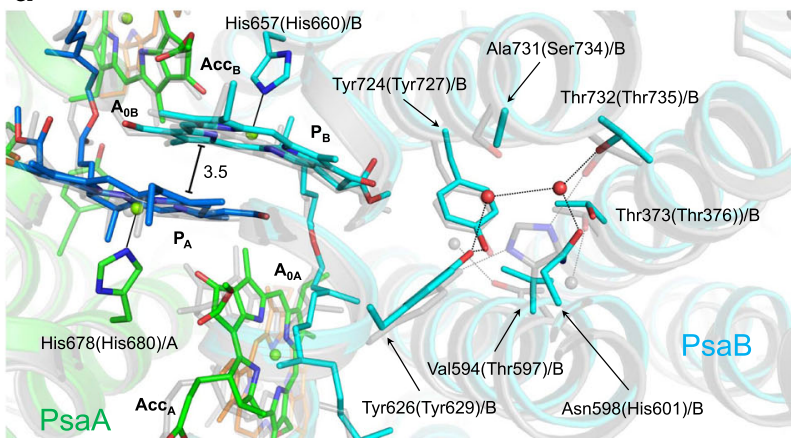

Fig. 3 Cofactors involved in the electron transfer reaction in A. marina PSI and their surrounding amino acid residues. a Arrangement of cofactors involved in the electron transfer reaction. Values on both sides represent the center-center distances (in $\AA$ ) between the cofactors. b Arrangement of P740 and its surrounding structure. View from the side of the membrane plane. $\mathbf{c} A s$ in $\mathbf{b}$, but view from the pseudo- $C_{2}$ axis. $\mathbf{d}$ Arrangement of the amino acid residues influencing $E_{m}\left(P_{A}\right)$ and $E_{m}\left(P_{B}\right)$. Superposition of the structural model of PSI from $A$. marina with that from T. elongatus (PDB code 1JBO). Transparent green and blue, PSI from A. marina; transparent gray, PSI from T. elongatus. Chlorophyll (Chl) $d$, green; Chl $d^{\prime}$, blue; water molecule, red; pheophytin (Pheo) a, orange, phylloquinone (PhyQ), yellow.

Chl $d^{\prime}$ and Chl $d$, respectively. Similarly, there were no amino acid residues and pigments capable of forming a hydrogen bond around the formyl group of $\mathrm{Acc}\left(\mathrm{Acc}_{\mathrm{A}}\right.$ and $\mathrm{Acc}_{\mathrm{B}}$; Fig. $\left.4 \mathrm{c}, \mathrm{d}\right)$. However, the orientations of the formyl group of $\mathrm{Acc}\left(\mathrm{Acc}_{\mathrm{A}}\right.$ and $\mathrm{Acc}_{\mathrm{B}}$ ) were altered by the hydrophobic environment caused by Trp (Trp585/B and Trp599/A) when compared with P740.

Previous studies have suggested that the primary electron acceptor $\mathrm{A}_{0}$ in A. marina PSI (Fig. 1a) is Chl $a$ (refs. ${ }^{13,41}$ ), in line with other species ${ }^{1,42}$. However, we found that there was no $\mathrm{Mg}^{2+}$-derived density at the center of the tetrapyrrole rings of $\mathrm{A}_{0 \mathrm{~A}}$ and $\mathrm{A}_{0 \mathrm{~B}}$, but rather a hole in the cryo-EM density map (Figs. $4 \mathrm{e}, \mathrm{f}$ and $5 \mathrm{a}$ ). This indicates, when combined with the result of pigment analysis (Supplementary Table 6 and Supplementary Fig. 19), that $\mathrm{A}_{0}$ is actually Pheo $a$, a derivative of Chl $a$. Furthermore, the position of Leu665/B (Supplementary Fig. 20b) ${ }^{43}$ pointing to $A_{0 B}$, supports Pheo $a$ as $\mathrm{A}_{0 \mathrm{~B}}$ in $A$. marina, because Leu cannot serve as a ligand to $\mathrm{Mg}^{2+}$ in Chl. Thus, surprisingly, $\mathrm{A}_{0 \mathrm{~A}}$ and $\mathrm{A}_{0 \mathrm{~B}}$ are assigned as Pheo $a$ in A. marina PSI (Figs. 1b, 3a, and 5a-d). The present study reveals that the axial amino acid residues at the $\mathrm{A}_{0 \mathrm{~A}}$ and $\mathrm{A}_{0 \mathrm{~B}}$ sites are Met686/A and Leu665/B, respectively. In comparison, the $\mathrm{A}_{0 \mathrm{~A}}$ and $\mathrm{A}_{0 \mathrm{~B}}$ sites in T. elongatus PSI are both occupied by $\mathrm{Chl} a$ molecules whose central $\mathrm{Mg}^{2+}$ ions are both coordinated by Met residues ${ }^{1,43}$. At present, it is unclear how the A. marina PSI selectively binds Pheo $a$ at the $\mathrm{A}_{0}$ sites instead of other pigments ( $\mathrm{Chl} a$ or $\mathrm{Chl} d$ ). However, the switch from Chl $a$ to Pheo $a$ in the $\mathrm{A}_{0 \mathrm{~A}}$ and $\mathrm{A}_{0 \mathrm{~B}}$ sites of $A$. marina PSI is probably not entirely dependent on the difference in its central ligand.

The amino acid residue nearest to $\mathrm{A}_{0 \mathrm{~A}}$ in $A$. marina is Met (Met686/A; Supplementary Fig. 20a) ${ }^{43}$ and the identity of close amino acid residues may modify the reduction potentials of the two $A_{0}$ sites and their absorption peak wavelength(s). Two mechanisms have been proposed for electron transfer in PSI reaction centers, one using both $\mathrm{A}$ - and $\mathrm{B}$-branches ${ }^{44,45}$, and the other using the A-branch preferentially ${ }^{46,47}$. Only the A-branch may be active in A. marina $\mathrm{PSI}^{43}$. Two previous studies have reported different mechanisms for the delocalization of the charge distribution in P740 (refs. ${ }^{48,49}$ ). Future theoretical studies using the A. marina PSI structure may throw more light on the details 

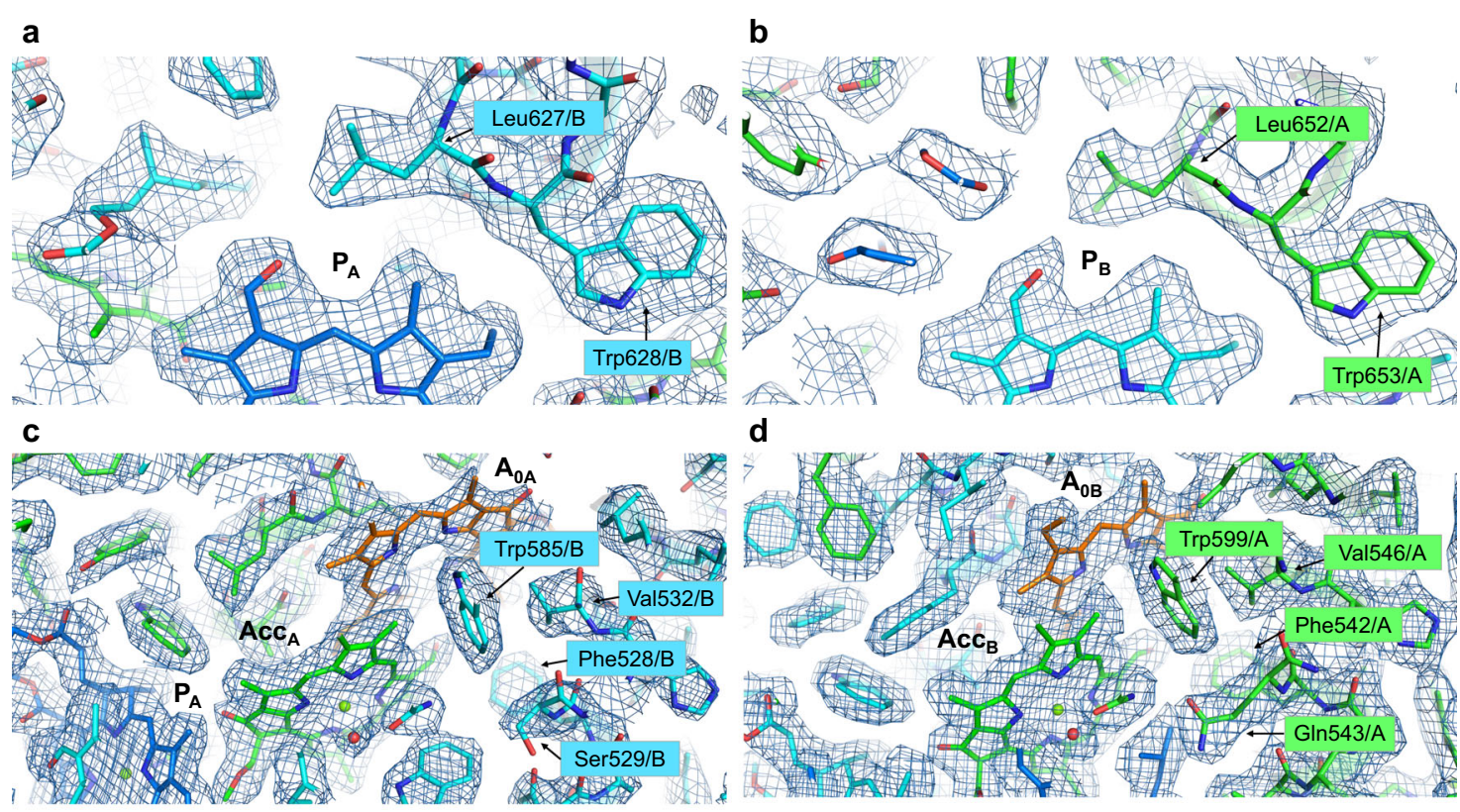

d
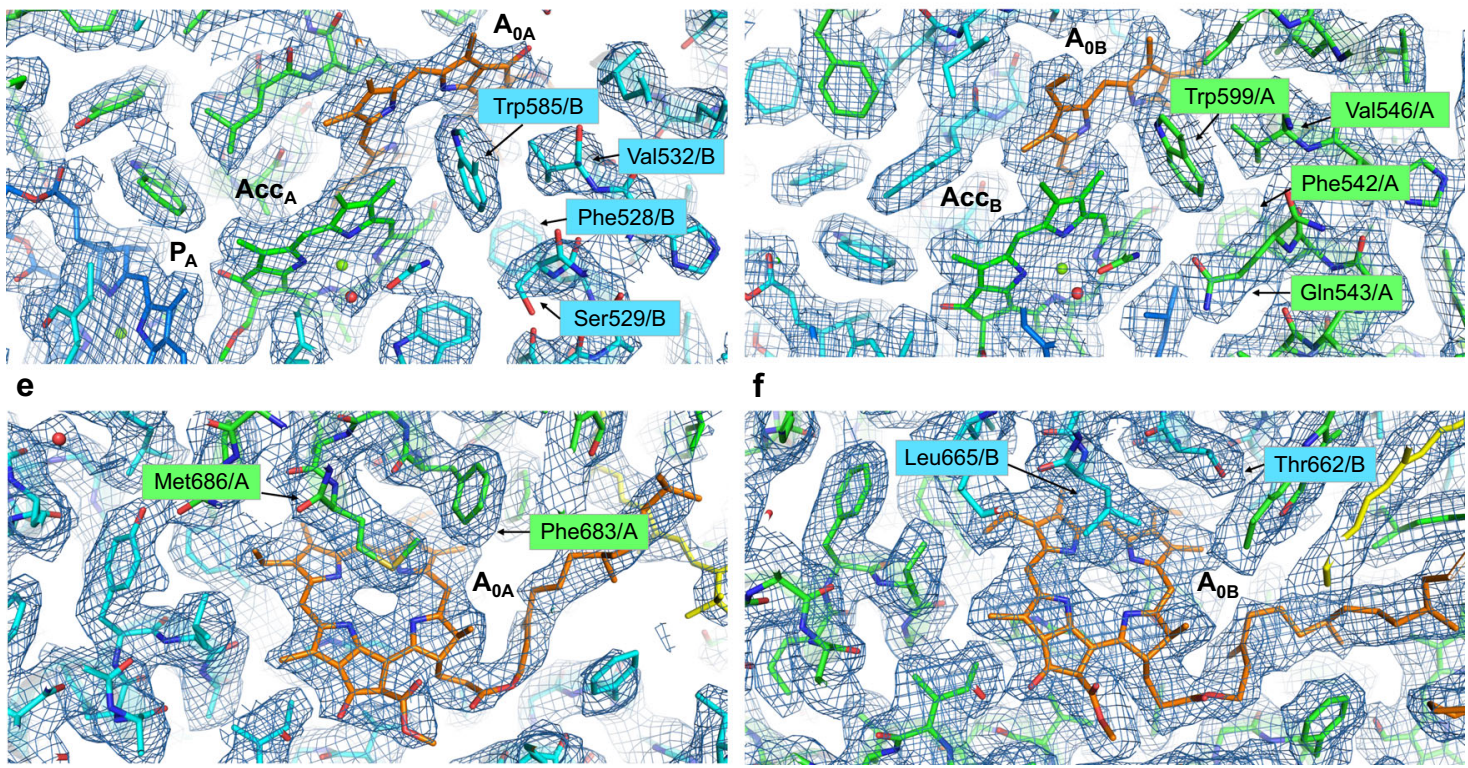

f

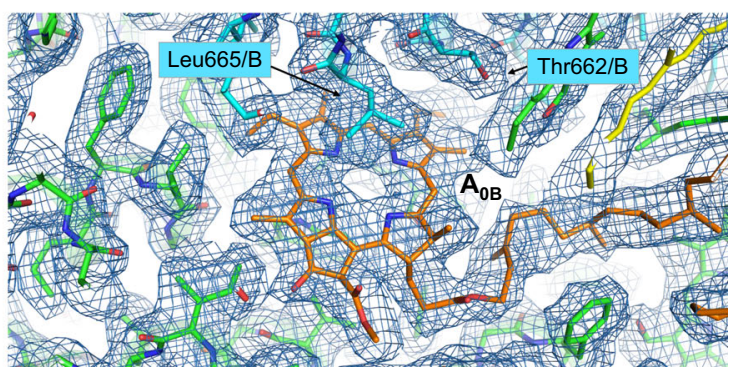

Fig. 4 Cryo-EM density maps around P740, Acc and $\mathbf{A}_{\mathbf{0}}$. Each map around P740 (a, b), Acc (c, d), and $A_{0}(\mathbf{e}$, $\mathbf{f})$ is shown in a mesh representation at 3.0 sigma contour level for $\mathbf{a}, \mathbf{b}, \mathbf{e}$, and $\mathbf{f}$, and 5.0 sigma for $\mathbf{c}$ and $\mathbf{d}$ for ease of recognition of the water molecule coordinating the $\mathrm{Mg}^{2+}$ of chlorin. Colors are the same as in Fig. 3.

of the electron transfer mechanism in relation to the $\mathrm{P}_{\mathrm{A}}{ }^{+}+$/ $\mathrm{P}_{\mathrm{B}} \cdot+$ ratio.

Why is Pheo $a$ the primary acceptor, $\mathrm{A}_{0}$, in A. marina PSI? According to the midpoint potential value $\left(E_{\mathrm{m}}\right)$, it seems reasonable for the $\mathrm{Chl} d$-driven PSI to use Pheo $a$ as $\mathrm{A}_{0}$, since the energy gap is sufficient for the primary electron transfer step as is estimated below. The $E_{\mathrm{m}}$ of P740 vs. the standard hydrogen electrode (SHE) is $439 \mathrm{mV}$ (refs. ${ }^{12,13,18,50}$ ), which is comparable with that of P700 (470 mV, ref. $\left.{ }^{51}\right)$. While the special pair P740, which are Chl $d / d^{\prime}$, use low-energy far-red light of $740 \mathrm{~nm}$ (1.68 $\mathrm{eV}$ ), it generates reducing power almost equivalent to that of the P700 (Chl $\left.a / a^{\prime}, 1.77 \mathrm{eV}\right)$ in plants and most cyanobacteria ${ }^{12}$. The produced reducing power of the excited state of $\mathrm{P} 740\left(\mathrm{P} 740^{*}\right)$ is weaker by $0.09 \mathrm{eV}$ than that of P700* (i.e., $1.77-1.68 \mathrm{eV}$ ). This could result in a slower electron transfer rate to $A_{0}$ and an increase in reverse reaction without a change in $E_{\mathrm{m}}$ of $\mathrm{A}_{0}$, due to the smaller driving force. It is reported that the rates of electron transfer from $\mathrm{P} 740^{*}$ to $\mathrm{A}_{0}$, and to PhyQ are actually comparable to those from P700* to $\mathrm{A}_{0}$ and to PhyQ ${ }^{41}$. Then, $E_{\mathrm{m}}$ of $\mathrm{A}_{0}$ has to change for a proper forward reaction. Because most of the amino acid residues around $\mathrm{A}_{0}$ in $A$. marina PSI are similar to those in T. elongatus, it is unlikely that the protein structure around $\mathrm{A}_{0}$ influences the $E_{\mathrm{m}}$ value. Therefore, we looked at the $E_{\mathrm{m}}$ value of the cofactor molecule itself, and obtained $E_{\mathrm{m}}$ (vs. SHE) values of purified Chl $a$, Chl $d$, and Pheo $a$ in acetonitrile of $-1100,-910$, and $-750 \mathrm{mV}$, respectively (Supplementary Fig. 21) ${ }^{51,52}$. The $E_{\mathrm{m}}$ of Pheo $a$ is the highest. Accordingly, Pheo $a$ as $\mathrm{A}_{0}$ should achieve the same electron transfer efficiency as the Chl $a$-type PSI. Then, reinvestigation of a possible effect on rate of the following $A_{0}$ to PhyQ (A1) step may be warranted as the charge recombination kinetics between $\mathrm{P}^{+} 40^{+}$and $\mathrm{A}_{1}{ }^{-}$has been reported to be comparable to those between $\mathrm{P} 700^{+}$and $\mathrm{A}_{1}{ }^{-}$of Chl $a$-type $\mathrm{PSI}^{50}$.

A. marina contains a limited but distinct amount of Chl $a$ (refs. $\left.{ }^{12,14,43}\right)$, and we found a small amount in the PSI (1-2 Chl $a$ per PSI monomer; Supplementary Table 6). It was once assumed that $\mathrm{A}_{0}$ is Chl $a$ (ref. ${ }^{41}$ ), but we now know this to be incorrect. One possible place for Chl $a$ is as the accessory Chls, $\mathrm{Acc}_{\mathrm{A}}$, and $\mathrm{Acc}_{\mathrm{B}}$ (Figs. 1 and 5). Unfortunately, the functional group containing C3 (ref. ${ }^{1}$ ) of Acc Chl could not be precisely defined from the cryo-EM density map. While quantum mechanical/molecular mechanical calculations show that the formyl group of Chl $d$ adopts two orientations (Supplementary Fig. 1c, d $)^{53}$, the oxygen atom of the formyl group on the Chl $d$ molecule in a vacuum is more stable when oriented toward the C5 $\mathrm{H}$ atom, suggesting the conformer in Supplementary Fig. 1c. In contrast, the vinyl group of $\mathrm{Chl} a$ can adopt either orientation. Therefore, Acc could not be conclusively identified as Chl $d$ or Chl $a$ at the present resolution, and we assigned Accs as Chl $d$ in this study. The $\mathrm{Mg}^{2+}$ in $\mathrm{Acc}_{\mathrm{A}}$ and $\mathrm{Acc}_{\mathrm{B}}$ are coordinated by water molecules forming hydrogen bonds with Asn588/B and Asn602/A, respectively (Fig. 5b, c). In T. elongatus PSI, the methyl ester groups of the Chl $a$ in $\mathrm{Acc}_{\mathrm{A}}$ and $\mathrm{Acc}_{\mathrm{B}}$ affect the charge and spin distributions on P700 (refs. ${ }^{40,54-56}$ ). In A. marina PSI, these distances were estimated to be $6.2 \AA$ $\left(\mathrm{Acc}_{\mathrm{A}}-\mathrm{P}_{\mathrm{A}}\right)$ and $6.6 \AA\left(\mathrm{Acc}_{\mathrm{B}}-\mathrm{P}_{\mathrm{B}}\right)$, respectively, in the present structure. 
a
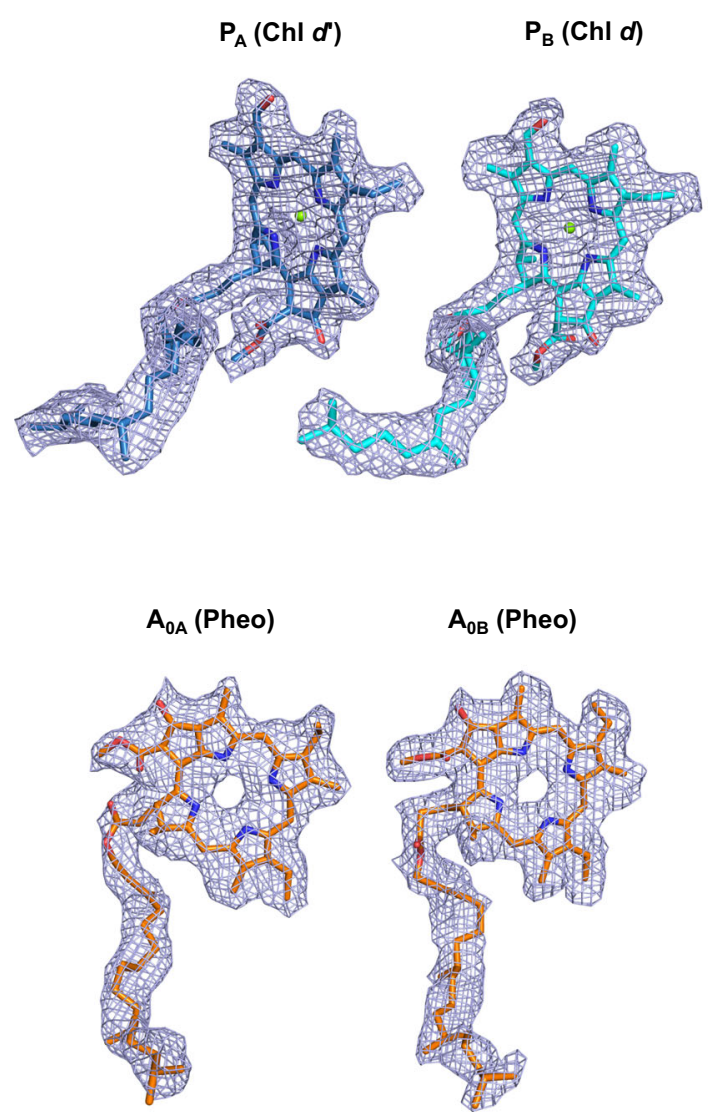

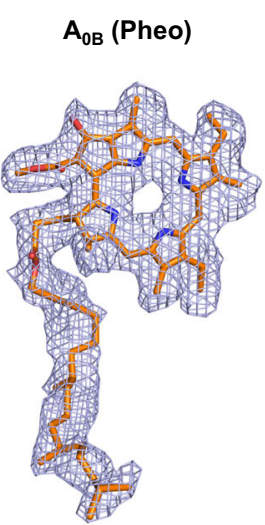

b

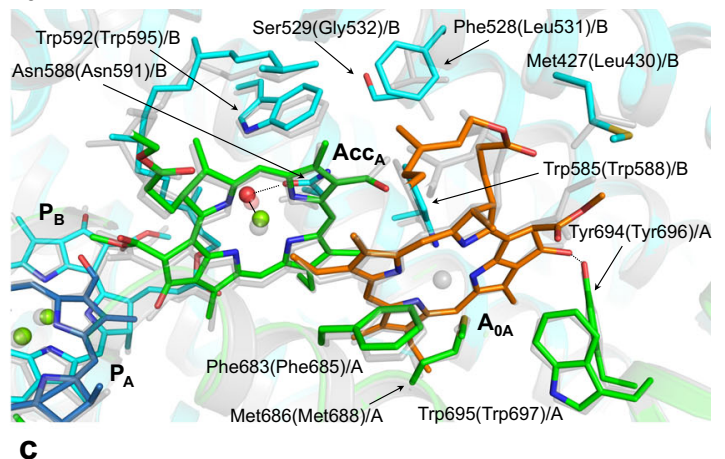

C

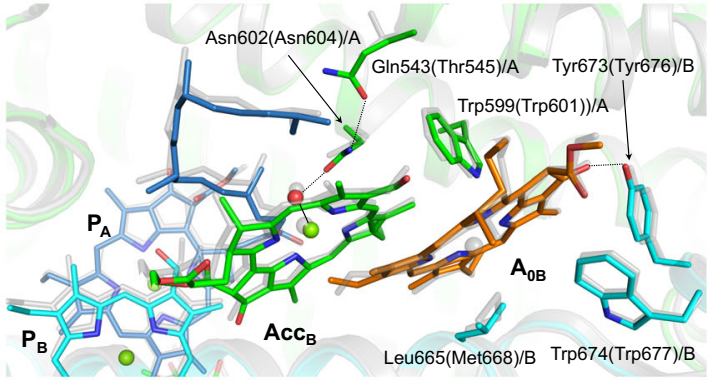

d

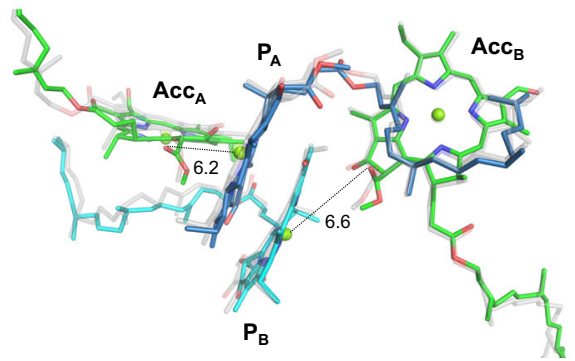

Fig. 5 Arrangement of $\mathbf{A}_{\mathbf{0}}, \mathbf{A c c}$, and the surrounding protein environment. The structural model of PSI from A. marina is superposed with that from T. elongatus (PDB code 1JBO). a Cryo-EM density map of $P_{A}, P_{B}, A_{O A}$, and $A_{O B}$. Each map is shown in a mesh representation at 1.0 sigma contour level. b Arrangement of $A_{O}, A c c$, and the surrounding protein environment. $\mathbf{c} A$ in $\mathbf{b}$, but view from the pseudo- $C_{2}$ axis. $\mathbf{d}$ Geometry of $P_{A} / P_{B}$ and $A c c\left(A c C_{A}\right.$ and $A\left(c_{B}\right) . C h l d$, green; Pheo, orange; water molecule, red. Colors are the same as in Fig. 3.

The amino acid residues surrounding PhyQ $\left(\mathrm{A}_{1 \mathrm{~A}}\right.$ and $\left.\mathrm{A}_{1 \mathrm{~B}}\right)$ are switched compared with those in T. elongatus PSI-Met720/A and Leu665/B in A. marina vs. Leu722/A and Met668/B in T. elongatus (Supplementary Fig. 22a, b). The arrangement of the phytol chain of $A_{1 B}$ is also different from that in other cyanobacteria (Supplementary Fig. 22b). These structural differences may suggest that the protein environment within $A$. marina PSI containing Chl $d$ is modified to support forward electron transfer by suppressing the reverse electron transfer from PhyQ to $\mathrm{A}_{0}$. However, cofactors $\mathrm{F}_{\mathrm{X}}, \mathrm{F}_{\mathrm{A}}$, and $\mathrm{F}_{\mathrm{B}}$ and their surrounding structures in A. marina PSI are nearly identical to those in other cyanobacteria (Fig. 3a and Supplementary Fig. 22). This indicates that the reduction potentials are likely at the same level, although the three $\mathrm{Fe} / \mathrm{S}$ clusters, $\mathrm{Fx}, \mathrm{F}_{\mathrm{A}}$, and $\mathrm{F}_{\mathrm{B}}$, transfer electrons from PhyQ $\left(A_{1}\right)$ to ferredoxin and the environment does not need to be conserved as long as the overall energy trajectory is downhill.

A. marina PSI is a trimer as the other cyanobacterial PSIs, but the amino acid sequence identity of each protein subunit is low, and, not unexpectedly, the divergence is most pronounced around the pigments specifically observed in A. marina PSI, for example Chl $d$, $a$-Car, and Pheo $a-\mathrm{A}_{0}$ (Supplementary Table 5). Again, the alterations must reflect optimization to drive efficient photochemistry utilizing low-energy light.

In conclusion, the structure and identification of electron carriers and key light-harvesting pigments provide a basis for understanding how low-energy far-red light is utilized by $A$. marina PSI. However, the full picture must wait for the structure of A. marina PSII, the system responsible for oxygen evolution through water-splitting, to be elucidated ${ }^{3}$.

\section{Methods}

Cell culture and thylakoid membrane preparation. A. marina was cultivated in artificial sea water Marine Art SF-1 (Osaka Yakken, Osaka, Japan) enriched with Daigo IMK (Nihon Pharmaceutical, Tokyo, Japan), using 7.5-L culture bottles (Rectangular Polycarbonate Clearboy, Nalgene, Rochester, NY) under continuous white light at $5-15 \mu \mathrm{moL}$ photons $\mathrm{m}^{-2} \mathrm{~s}^{-1}$ and $23^{\circ} \mathrm{C}$ with continuous air-bubbling for 25-28 days. Cells were harvested by centrifugation at $7800 \times g$ for $10 \mathrm{~min}$, suspended in buffer A containing $50 \mathrm{mM} \mathrm{2-(N-morpholino)ethanesulfonic} \mathrm{acid}$ (MES)- $\mathrm{NaOH}$ (pH 6.5), $5 \mathrm{mM} \mathrm{CaCl}_{2}, 10 \mathrm{mM} \mathrm{MgCl} 2,1.0 \mathrm{M}$ betaine, and $5 \%$ (w/v) glycerol, and stored frozen until use. The cells were broken at $1.5 \mathrm{mg} \mathrm{Chl} d \mathrm{~mL}^{-1}$ with the same volume of glass beads $\left(0.2 \mathrm{~mm}\right.$ diameter) at $0^{\circ} \mathrm{C}$ by 24 cycles of $10^{-\mathrm{s}}$ breaking, and 2-min cooling in the presence of DNase I $\left(0.5 \mu \mathrm{gL}^{-1}\right.$; Sigma, St. Louis, MO) and a protease inhibitor mixture $(280 \mu \mathrm{L}$ per $100 \mathrm{~mL}$ cell suspension; Sigma). Thylakoid membranes were collected by centrifuging the broken cells at $31,000 \times g$ for $10 \mathrm{~min}$. The resulting pellets of thylakoid membrane were resuspended in buffer $A$.

Preparation of PSI trimer from A. marina membrane. Prior to the purification of PSI complex, solubilization of thylakoids was tested using 13 detergents that are frequently used to solubilize membrane protein complexes: $n$-decyl- $\beta$-D-maltopyranoside (DM), $n$-undecyl- $\beta$-D-maltopyranoside (UDM), $n$-dodecyl- $\beta$-D-maltopyranoside, $n$-tetradecyl- $\beta$-D-maltopyranoside, $n$-dodecyl- $\alpha$-D-maltopyranoside, $n$-octyl- $\beta$-D-glucopyranoside, 6-cyclohexyl-1- $\beta$-D-maltopyranoside, $N$ - 
lauroylsarcosine, $n$-decyl- $\beta$-D-maltopyratose neopentyl glycol (LMNG), decyl maltose neopentyl glycol, sodium cholate, and digitonin. Because the first three detergents had almost the same solubilization efficiency, UDM was selected. DM was used in sucrose density gradient ultracentrifugation because it was the best of the three detergents for maintaining the stability of the purified PSI trimer (Supplementary Fig. 5).

Thylakoid membranes were solubilized with $1.0 \%(\mathrm{w} / \mathrm{v}) \mathrm{UDM}$ at $1 \mathrm{mg} \mathrm{Chl}$ $d \mathrm{~mL}^{-1}$ on ice for $10 \mathrm{~min}$. The extracts were separated from insoluble membranes by centrifugation at $75,600 \times g$ for $10 \mathrm{~min}$, and layered onto a stepwise sucrose gradient $(1.4,1.2,1.1,1.05,1.0,0.9,0.8$, and $0.7 \mathrm{M}$ sucrose in buffer A supplemented with $0.2 \%(\mathrm{w} / \mathrm{v}) \mathrm{DM})$ for ultracentrifugation at $100,000 \times \mathrm{g}$ for $20 \mathrm{~h}$. After centrifugation, fractionation was performed from bottom to top, and the protein content of each fraction was investigated by blue native-polyacrylamide gel electrophoresis using linear gradient gels of 3-12\% polyacrylamide (NativePage Bis-Tris; Thermo Fisher Scientific, Waltham, MA; Supplementary Fig. 4). Fractions exhibiting a band of PSI trimer of $\sim 720 \mathrm{kDa}$, corresponding to sucrose concentrations of 1.05-1.0 M, were collected.

For electron microscopic analysis, the PSI trimer in the above fractions was washed using Amicon centrifugal filters (pore size 100,000 Da) with buffer A supplemented with $0.002 \%$ LMNG, and applied to a second stepwise sucrose gradient ultracentrifugation consisting of 1.4, 1.2, 1.1, 1.05, 1.0, 0.9, 0.8 , and $0.7 \mathrm{M}$ sucrose in buffer A supplemented with $0.002 \%$ LMNG. LMNG was used for electron microscopic analysis because of its low critical micellar concentration.

For biochemical analyses, the fractions obtained after the first sucrose density gradient ultracentrifugation were applied to an anion exchange column (HiLoad 16/10 Q Sepharose HP) and eluted with a linear gradient of $0-600 \mathrm{mM} \mathrm{KCl}$.

Polypeptide analysis. Polypeptides denatured with or without dithiothreitol were separated on a gel containing $0.1 \%(\mathrm{w} / \mathrm{v})$ sodium dodecyl sulfate, $6 \mathrm{M}$ urea, $0.6 \mathrm{M}$ Tris, $0.13 \mathrm{M}$ MES (pH9.0), and 16-22\% (w/v) acrylamide ${ }^{57}$, and then stained with Coomassie Blue (Supplementary Fig. 6). Separated polypeptides were identified by mass spectrometry analysis after in-gel digestion by trypsin (Supplementary Table 2).

Spectroscopic analysis. $\mathrm{Chl} a$ and $\mathrm{Chl} d$ concentrations were determined after extraction by methanol using methanol as the solvent and a UV-2700 spectrophotometer (Shimadzu; Kyoto, Japan) with a slit-width of $1 \mathrm{~nm}$ at $25^{\circ} \mathrm{C}$. The mass extinction coefficients for $\mathrm{Chl} a$ and $\mathrm{Chl} d$ was 79.95 (at $665.2 \mathrm{~nm})^{58}$ and 71.11 (at $697.0 \mathrm{~nm})^{59} \mathrm{~L} \mathrm{~g}^{-1} \mathrm{~cm}^{-1}$, respectively, Absorption spectra of the PSI trimers were measured at $25^{\circ} \mathrm{C}$ using an UV-2700 spectrophotometer (Supplementary Fig. 6). For cell suspensions, an integrating sphere, model ISR-2600, was used with the UV2700 spectrophotometer (Supplementary Figs. 2 and 6). Absorption spectra at $77 \mathrm{~K}$ were measured using an MPS 2000 spectrophotometer equipped with a lowtemperature measurement unit, LTS-2000 (Shimadzu; Supplementary Fig. 7).

Pigment analysis. Photosynthetic pigments and quinones were extracted in methanol and quantified using reversed-phase high-performance liquid chromatography carried out, using an LC-20AT with a CBM-20A system controller (Shimadzu), equipped with a Kinetex C18 column $(5 \mu \mathrm{m}, 250 \times 4.60 \mathrm{~mm}$, $100 \AA$ A ; Phenomenex, Torrance, CA), equilibrated with $80 \%$ methanol containing $20 \mathrm{mM}$ ammonium acetate (solvent A). Pigments were eluted at a flow rate of $0.8 \mathrm{~mL} \mathrm{~min}-1$ over a period of $60 \mathrm{~min}$ with a gradient of $0-100 \%$ of ethyl acetate/ methanol $(30 / 70 \mathrm{v} / \mathrm{v} \text {, solvent } \mathrm{B})^{60}$; the ratio of solvent $\mathrm{B}$ was increased to $30 \%$ in $4 \mathrm{~min}$ and then to $100 \%$ over a further $36 \mathrm{~min}$, and then, keeping the ratio for another $20 \mathrm{~min}$. The elution pattern of the pigments was detected using a Shimadzu photodiode array detector, SPD-M20A, with Shimadzu LabSolutions (ver. 5.82) analysis software at $430 \mathrm{~nm}$ for photosynthetic pigments (Supplementary Fig. 19) and $270 \mathrm{~nm}$ for PhyQ. Standard curves for Chl $a$ and Chl $d$ were obtained after quantification of $\mathrm{Chl} a$ from a cyanobacterium, Synechocystis sp. PCC 6803, and Chl $d$ from A. marina as described above. The standard curve for Chl $d$ was also used for Chl $d^{\prime}$. A standard curve for Pheo $a$ was made using Pheo $a$ obtained by acidification of quantified $\mathrm{Chl} a$. PhyQ (vitamin $\mathrm{K}_{1}$ ) was obtained from Wako Pure Chemicals (Osaka, Japan). Zeaxanthin and $\alpha$ - and $\beta$-carotene were purchased from the VKI Water Quality Institute (Hørsholm, Denmark). PhyQ bound to PSI trimer was quantified after the removal of $\mathrm{Chl} a$ contribution at almost the same retention time by acidification ${ }^{61}$

DNA sequencing. Genes $p s a A$ and $p s a B$ were partially sequenced as follows (Supplementary Fig. 20). Pairs of primers (Supplementary Table 7) for $p s a A$ (GTACAACTGCATCTCAATTG and CTATCCTAATGCGAGAATTC) and $p s a B$ (CCTTGCCTTCTTCTGGATGC and TTAGCCGAGAGGAGCTGTTG) were used to amplify part of the genes by PCR, using genomic DNA as the template. Then, the DNA sequence of the amplified, purified DNA fragment was analyzed using BigDye Terminator ver. 3.1 (Applied Biosystems; Foster City, CA), using the same primers.

Cryo-EM sample preparation and data collection. Three microliters of purified PSI (0.4 mg Chl $d \mathrm{~mL}^{-1}$ in a buffer containing $50 \mathrm{mM}$ MES-NaOH (pH 6.5),
$5 \mathrm{mM} \mathrm{CaCl}_{2}, 10 \mathrm{mM} \mathrm{MgCl}_{2}$, and $0.002 \% \mathrm{LMNG}$ ) were applied to a holey carbon grid (Quantifoil R1.2/1.3, Cu, 200 mesh grids, Microtools GmbH, Berlin, Germany) that had been pretreated by gold-sputtering 62,63 and glow-discharging (JEC-3000FC, JEOL, Japan). The grid was blotted with filter paper for $4 \mathrm{~s}$, then immediately plunge-frozen in condensed ethane using an FEI Vitrobot Mark IV (Thermo Fisher Scientific, Waltham, MA, USA) under $100 \%$ humidity at $4{ }^{\circ} \mathrm{C}$. The frozen grids were then introduced into a CRYO ARM 300 electron microscope (JEOL, Tokyo, Japan) equipped with a cold-field emission gun, after which inelastic scattered electrons were removed using an in-column type energy filter with an energy slit of $20 \mathrm{eV}$. Dose fractionated images were recorded on a $\mathrm{K} 2$ summit camera in counting mode with a nominal magnification of $60,000 \times$, which corresponded to a physical pixel size of $0.823 \AA$. All image data were collected with a JEOL Automatic Data Acquisition System (JADAS) ${ }^{64}$ with a dose rate of $8.57 \mathrm{e}^{-} \AA^{-2} \mathrm{~s}^{-1}, 10 \mathrm{~s}$ exposures, $0.2 \mathrm{~s}$ per frame, and a nominal defocus range of -0.8 to $-1.8 \mu \mathrm{m}$. In total, we collected 3225 (data 1) and 4346 movie stacks (data 2) from two independent sample preparations.

Cryo-EM image processing. Cryo-EM movie stacks were grouped into ten separate optics groups based on time of data collection. Drift corrected and doseweighted summation of movie frames was performed using MotionCor2 (version 1.1.3 $)^{65}$, and contrast transfer function (CTF) parameters were estimated from the correct images with CTFFIND4 (version 4.1.10) ${ }^{66}$. Based on Thon-ring patterns, 4237 images from data 1 and 2 were selected for particle picking. Good PSI particles were manually selected and subjected to reference-free two-dimensional (2D) classification with RELION-3.1beta. Next, homogeneous 2D class averages were used as templates for reference-based auto-picking, and a total of 774,416 particles were extracted with a pixel size of $1.646 \AA$ and a box size of 220 pixels for further 2D classification. Good 2D class averages contained 242,550 particles, which were passed to three-dimensional (3D) classification with a de novo initial model constructed using cryoSPARC (version 2.12.0), and a well-aligned and symmetrical 3D class was reconstructed from 86,509 particles. The particles in the best $3 \mathrm{D}$ class were rescaled and re-extracted with a pixel size of $1.08 \AA$ and a box size of 330 pixels, and $3 \mathrm{D}$ refinement with threefold symmetry enforcement and postprocessing yielded a $3.3 \AA$ resolution map based on the gold standard Fourier shell correlation criterion. Particles in the cryo-EM density map were then subjected to Bayesian polishing for correction of particle-based beam-induced motion and CTF refinement of particle-based defoci and optics-group-based high-order astigmatisms. Finally, 86,419 particles were selected by excluding those showing unrealistic defocus values and then subjected to $3 \mathrm{D}$ refinement and post-processing again, which yielded a $2.59 \AA$ resolution map with C3 symmetrization. After beam tilt estimation and correction for every micrograph, 3D refinement and postprocessing reached $2.58 \AA$ and $2.97 \AA$ resolution structures with and without C3 symmetrization, respectively. $B$-factor values for map sharpening were estimated as -92.82 and $-77.95 \AA^{2}$, respectively. For further details, see Supplementary Figs. 9-11, and Supplementary Tables 3 and 4.

Model building and refinement. An initial model of the PSI monomer from A. marina was built using homology modeling in MODELLER (version 9.23$)^{67}$ by referring to the structure of the PSI monomer from T. elongatus (PDB code: 1JB0) The identity of each amino acid in subunit proteins in A. marina PSI was determined using amino acid sequences obtained from UniProt (https://www.uniprot. org/). The resulting homology model of the PSI monomer was fitted to the whole cryo-EM density map (C3 map), using a "fit in map" program in UCSF Chimera (version 1.13) ${ }^{6}$, and an initial model of the trimeric form of PSI from A. marina was created. Next, the created PSI trimer model was fitted to the cryo-EM density map using a molecular dynamics simulation program (CryoFit) and simulated annealing in Phenix ${ }^{69,70}$. Each model of the three monomers in the PSI trimer was refined separately, and the refined model was modified using COOT $^{71}$ manually to fit the cryo-EM density map. Subsequently, the obtained structure model was finally refined using refmac5 (ref. ${ }^{72}$; Supplementary Fig. 12). Refinement statistics of the refined model were obtained using a comprehensive validation program in Phenix.

The cofactors such as $\mathrm{Chl} d, \mathrm{Chl} d^{\prime}, \mathrm{Chl} a$, Pheo $a$, PhyQ, iron-sulfur clusters, $\alpha$ Car, MGDG, and PG were identified and refined using geometry restraint information, as described in the following section. For $\mathrm{A}_{0}\left(\mathrm{~A}_{0 \mathrm{~A}}\right.$ and $\left.\mathrm{A}_{0 \mathrm{~B}}\right)$ in $A$. marina PSI, a cryo-EM density of $\mathrm{Mg}^{2+}$ of Chl could not be identified unambiguously, and thus the two $\mathrm{A}_{0}$ were identified as Pheo. Validation between the refined model and the cryo-EM density local resolution map was assessed using Phenix, ModelZ ${ }^{73}$, and $\mathrm{MapQ}^{74}$. The refinement statistics of the models are summarized in Supplementary Table 1. Structure figures were generated and rendered with PyMOL and Chimera ${ }^{68,75}$.

Identification of cofactors and their geometry restraint information. The restraints needed for the pigments in PSI from A. marina were generated by Electronic Ligand Bond Builder and Optimization Workbench (eLBOW) ${ }^{76}$ because the information was not registered in the restraints library in Phenix. The restraint information for $\mathrm{Chl} d$ was created from the model of $\mathrm{Chl} d$ identified in a highresolution structure (PDB code: $2 \mathrm{X} 1 \mathrm{Z})^{77}$, and information for $\mathrm{Chl} d^{\prime}$, an epimer of $\mathrm{Chl} d$, was created with reference to the structures of $\mathrm{Chl} d$ and Chl $a^{\prime}$, an epimer of 
$\mathrm{Chl} a$ (PDB codes: 1JBO and 2X1Z). The orientation of the C3-formyl group of $\mathrm{Chl}$ $d^{\prime}$ was determined based on that of $\mathrm{Chl} d\left(\mathrm{ref} .{ }^{77}\right.$ ). Only a small amount of $\mathrm{Chl} a$ was present in the sample, and so we assigned the Chls of A. marina PSI as Chl $d$ in this study even though it is not possible to distinguish between Chl $d$ and Chl $a$ at 2.58 A resolution.

The PSI trimer from A. marina contained $\alpha$-Car instead of $\beta$-carotene ( $\beta$-Car). The $\alpha$-Car molecule possesses two rings-known as $\beta$ - and $\varepsilon$-rings-at each end, while the $\beta$-Car molecule possesses two $\beta$-rings. Although the restraint information for $\alpha$-Car was created using eLBOW, as for the other cofactors, $\alpha$-Car was registered as an unknown ligand (three-letter code: UNL) in this study because it is hard to distinguish between $\beta$ - and $\varepsilon$-rings from the present cryo-EM density map. Restraint information for Pheo, iron-sulfur clusters, MGDG (three-letter code: LMG), and PG (three-letter code: LHG) was created with reference to highresolution structures (PDB codes: 1 JB0 and $3 \mathrm{WU} 2)$.

Identification of water molecules. Water molecules were identified manually from the cryo-EM density map at $>2.0$ root-mean-square level using COOT. Eighty-four water molecules were found in the refined structure model. Most of the water molecules were identified as ligands to the $\mathrm{Mg}^{2+}$ in $\mathrm{Chl} d$.

Reporting summary. Further information on research design is available in the Nature Research Reporting Summary linked to this article.

\section{Data availability}

Atomic coordinates and cryo-EM maps for the reported structure of Acaryochloris marina PSI have been deposited in the Protein Data Bank under accession codes 7COY, and in the Electron Microscopy Data Bank under accession codes EMD-30420, respectively. Other data are available from the corresponding authors upon reasonable request. Source data are provided with this paper.

Received: 30 July 2020; Accepted: 18 March 2021; Published online: 20 April 2021

\section{References}

1. Jordan, P. et al. Three-dimensional structure of cyanobaoterial photosystem I at $2.5 \AA$ resolution. Nature 411, 909-917 (2001).

2. Umena, Y., Kawakami, K., Shen, J. R. \& Kamiya, N. Crystal structure of oxygen-evolving photosystem II at a resolution of 1.9 . Nature 473, 55-60 (2011).

3. Nelson, N. \& Junge, W. Structure and energy transfer in photosystems of oxygenic photosynthesis. Annu. Rev. Biochem. 84, 659-683 (2015).

4. Miyashita, H. et al. Chlorophyll $d$ as a major pigment. Nature 383, 402 (1996).

5. Chen, M. et al. A red-shifted chlorophyll. Science 329, 1318-1319 (2010).

6. Gan, F. et al. Extensive remodeling of a cyanobacterial photosynthetic apparatus in far-red light. Science 345, 1312-1317 (2014).

7. Miyashita, H. Discovery of chlorophyll $d$ in Acaryochloris marina and chlorophyll $f$ in a unicellular cyanobacterium, strain $\mathrm{KC1}$, isolated from Lake Biwa. J. Phys. Chem. Biophys. 4, 149 (2014).

8. Kosugi, M., Kashino, Y. \& Kazuhiko, S. Comparative analysis of light response curves of Ramalina yasudae and freshly isolated Trebouxia sp. revealed the presence of intrinsic protection mechanisms independent of upper cortex for the photosynthetic system of algal symbionts in lichen. Lichenology 9, 1-10 (2010).

9. Ohashi, S. et al. Unique photosystems in Acaryochloris marina. Photosynth. Res. 98, 141-149 (2008).

10. Swingley, W. D. et al. Niche adaptation and genome expansion in the chlorophyll $d$-producing cyanobacterium Acaryochloris marina. Proc. Natl. Acad. Sci. USA 105, 2005-2010 (2008).

11. Itoh, S. et al. Function of chlorophyll $d$ in reaction centers of photosystems I and II of the oxygenic photosynthesis of Acaryochloris marina. Biochemistry 16, 12473-12481 (2007).

12. Tomo, T. et al. Characterization of highly purified photosystem I complexes from the chlorophyll $d$-dominated cyanobacterium Acaryochloris marina MBIC 11017. J. Biol. Chem. 283, 18198-18209 (2008).

13. Itoh, S. et al. Function of chlorophyll $d$ in reaction centers of photosystems I and II of the oxygenic photosynthesis of Acaryochloris marina. Biochemistry 46, 12473-12481 (2007).

14. $\mathrm{Hu}, \mathrm{Q}$. et al. A photosystem I reaction center driven by chlorophyll $d$ in oxygenic photosynthesis. Proc. Natl. Acad. Sci. USA 95, 13319-13323 (1998).

15. Akiyama, M. et al. Stoichiometries of chlorophyll $d^{\prime} / \mathrm{PS} 1$ and chlorophyll $a /$ PSII in a chlorophyll $d$-dominated cyanobacterium Acaryochloris marina, Jpn. J. Phycol. 52, 67-72 (2004).

16. Hastings, G. Time-resolved step-scan Fourier transform infrared and visible absorption difference spectroscopy for the study of photosystem I. Appl. Spectrosc. 55, 894-900 (2001).
17. Kobayashi, M. et al. Chlorophyll $a^{\prime} / \mathrm{P}-700$ and pheophytin $a / \mathrm{P}-$ 680 stoichiometries in higher plants and cyanobacteria determined by HPLC analysis. Biochim. Biophys. Acta Bioenerg. 936, 81-89 (1988).

18. Bailleul, B. et al. The thermodynamics and kinetics of electron transfer between cytochrome $b 6 f$ and photosystem I in the chlorophyll $d$-dominated cyanobacterium, Acaryochloris marina. J. Biol. Chem. 283, 25218-25226 (2008).

19. Kato, K. et al. Structural basis for the adaptation and function of chlorophyll $f$ in photosystem I. Nat. Commun. 11, 238 (2020).

20. Kaucikas, M., Nürnberg, D., Dorlhiac, G., Rutherford, A. W. \& van Thor, J. J. Femtosecond visible transient absorption Sspectroscopy of chlorophyll f-containing photosystem I. Biophys. J. 112, 234-249 (2017).

21. Nürnberg, D. J. et al. Photochemistry beyond the red limit in chlorophyll $f$-containing photosystems. Science 360, 1210-1213 (2018).

22. Gisriel, C. et al. The structure of photosystem I acclimated to far-red light illuminates an ecologically important acclimation process in photosynthesis Sci. Adv. 6, eaay6415 (2020)

23. Gisriel, C. J., Wang, J., Brudvig, G. W. \& Bryant, D. A. Opportunities and challenges for assigning cofactors in cryo-EM density maps of chlorophyllcontaining proteins. Commun. Biol. 3, 408 (2020).

24. Kurashov, V. et al. Energy transfer from chlorophyll $f$ to the trapping center in naturally occurring and engineered Photosystem I complexes. Photosynth. Res. 141, 151-163 (2019).

25. Cherepanov, D. A. et al. Evidence that chlorophyll $f$ functions solely as an antenna pigment in far-red-light photosystem I from Fischerella thermalis PCC 7521. Biochim. Biophys. Acta Bioenerg. 1861, 148184 (2020).

26. Judd, M. et al. The primary donor of far-red photosystem II: $\mathrm{Chl}_{\mathrm{D} 1}$ or $\mathrm{P}_{\mathrm{D} 2}$ ? Biochim. Biophys. Acta Bioenerg. 1861, 148248 (2020)

27. Schmitt, F. J. et al. Photosynthesis supported by a chlorophyll $f$-dependent, entropy-driven uphill energy transfer in Halomicronema hongdechloris cells adapted to far-red light. Photosynth. Res. 139, 185-201 (2019).

28. Mazor, Y., Borovikova, A. \& Nelson, N. The structure of plant photosystem I super-complex at $2.8 \AA$ resolution. Elife 4, e07433 (2015).

29. Qin, X., Suga, M., Kuang, T. \& Shen, J. R. Structural basis for energy transfer pathways in the plant PSI-LHCI supercomplex. Science 348, 989-995 (2015).

30. Qin, X. et al. Structure of a green algal photosystem I in complex with a large number of light-harvesting complex I subunits. Nat. Plants 5, 263-272 (2019).

31. Suga, M. et al. Structure of the green algal photosystem I supercomplex with a decameric light-harvesting complex I. Nat. Plants 5, 626-636 (2019)

32. $\mathrm{Pi}, \mathrm{X}$. et al. Unique organization of photosystem I-light-harvesting supercomplex revealed by cryo-EM from a red alga. Proc. Natl. Acad. Sci. USA 115, 4423-4428 (2018).

33. Nagao, R. et al. Structural basis for assembly and function of a diatom photosystem I-light-harvesting supercomplex. Nat. Commun. 11, 2481 (2020).

34. Kato, K. et al. Structure of a cyanobacterial photosystem I tetramer revealed by cryo-electron microscopy. Nat. Commun. 10, 4929 (2019).

35. Malavath, T., Caspy, I., Netzer-El, S. Y., Klaiman, D. \& Nelson, N. Structure and function of wild-type and subunit-depleted photosystem I in Synechocystis. Biochim. Biophys. Acta Bioenerg. 1859, 645-654 (2018).

36. Gisriel, C. et al. Structure of a symmetric photosynthetic reaction centerphotosystem. Science 357, 1021-1025 (2017).

37. Adolphs, J., Müh, F., Madjet, M. E. A., Am Busch, M. S. \& Renger, T. Structurebased calculations of optical spectra of photosystem I suggest an asymmetric light-harvesting process. J. Am. Chem. Soc. 132, 3331-3343 (2010).

38. Kölsch, A. et al. Current limits of structural biology: the transient interaction between cytochrome $c$ and photosystem I. Curr. Res. Struct. Biol. 2, 171-179 (2020).

39. Webber, A. N. et al. Site-directed mutations affecting the spectroscopic characteristics and midpoint potential of the primary donor in photosystem I. Biochemistry 35, 12857-12863 (1996)

40. Saito, K. \& Ishikita, H. Cationic state distribution over the P700 chlorophyll pair in photosystem I. Biophys. J. 101, 2018-2025 (2011).

41. Kumazaki, S., Abiko, K., Ikegami, I., Iwaki, M. \& Itoh, S. Energy equilibration and primary charge separation in chlorophyll $d$-based photosystem I reaction center isolated from Acaryochloris marina. FEBS Lett. 530, 153-157 (2002).

42. Akiyama, M. et al. Quest for minor but key chlorophyll molecules in photosynthetic reaction centers - Unusual pigment composition in the reaction centers of the chlorophyll $d$-dominated cyanobacterium Acaryochloris marina. Photosynth. Res. 74, 97-107 (2002).

43. Itoh, S. et al. in Photosynthesis. Energy from the Sun, 93-96 (Springer, 2008).

44. Guergova-Kuras, M., Boudreaux, B., Joliot, A., Joliot, P. \& Redding, K. Evidence for two active branches for electron transfer in photosystem I. Proc. Natl. Acad. Sci. USA 98, 4437-4442 (2001).

45. Ramesh, V. M., Gibasiewicz, K., Lin, S., Bingham, S. E. \& Webber, A. N. Bidirectional electron transfer in photosystem I: accumulation of $\mathrm{A}_{0}-$ in Aside or B-side mutants of the axial ligand to chlorophyll $\mathrm{A}_{0}$. Biochemistry 43 , 1369-1375 (2004).

46. $\mathrm{Xu}, \mathrm{W}$. et al. Electron transfer in cyanobacterial photosystem I. I. Physiological and spectroscopic characterization of site-directed mutants in a putative 
electron transfer pathway from $\mathrm{A}_{0}$ through $\mathrm{A}_{1}$ to Fx. J. Biol. Chem. 278, 27864-27875 (2003).

47. Cohen, R. O. et al. Evidence for asymmetric electron transfer in cyanobacterial photosystem I: Analysis of a methionine-to-leucine mutation of the ligand to the primary electron acceptor $A_{0}$. Biochemistry 43, 4741-4754 (2004).

48. Mino, H. et al. Proton ENDOR study of the primary donor $\mathrm{P} 740^{+}$, a special pair of chlorophyll $d$ in photosystem I reaction center of Acaryochloris marina. Chem. Phys. Lett. 411, 262-266 (2005).

49. Hastings, G. \& Wang, R. Vibrational mode frequency calculations of chlorophyll- $d$ for assessing (P740+-P740) FTIR difference spectra obtained using photosystem I particles from Acaryochloris marina. Photosynth. Res. 95, 55-62 (2008).

50. Schenderlein, M., Çetin, M., Barber, J., Telfer, A. \& Schlodder, E. Spectroscopic studies of the chlorophyll $d$ containing photosystem I from the cyanobacterium, Acaryochloris marina. Biochim. Biophys. Acta Bioenerg. 1777, 1400-1408 (2008).

51. Kobayashi, M. et al. in Photosynthesis (ed. Dubinsky, Z.) Ch. 3 (InTech, 2013).

52. Komatsu, H. et al. Physicochemical properties of divinyl chlorophylls $a, a^{\prime}$ and divinyl pheophytin $a$ compared with those of monovinyl derivatives. Photomed. Photobiol. 36, 59-69 (2014).

53. Saito, K., Shen, J. R. \& Ishikita, H. Cationic state distribution over the chlorophyll $d$-containing $\mathrm{P}_{\mathrm{D} 1} / \mathrm{P}_{\mathrm{D} 2}$ pair in photosystem II. Biochim. Biophys. Acta Bioenerg. 1817, 1191-1195 (2012).

54. Breton, J., Nabedryk, E. \& Leibl, W. FTIR study of the primary electron donor of photosystem I (P700) revealing delocalization of the charge in $\mathrm{P}^{2} 00^{+}$and localization of the triplet character in ${ }^{3}$ P700. Biochemistry 38, 11585-11592 (1999).

55. Davis, I. H., Heathcote, P., MacLachlan, D. J. \& Evans, M. C. W. Modulation analysis of the electron spin echo signals of in vivo oxidised primary donor ${ }^{14} \mathrm{~N}$ chlorophyll centres in bacterial, P870 and P960, and plant photosystem I, P700, reaction centres. Biochim. Biophys. Acta Bioenerg. 1143, 183-189 (1993).

56. Käss, H., Fromme, P., Witt, H. T. \& Lubitz, W. Orientation and electronic structure of the primary donor radical cation $\mathrm{P} 00^{+}$in photosystem I: a single crystals EPR and ENDOR study. J. Phys. Chem. B 105, 1225-1239 (2001).

57. Kashino, Y., Koike, H. \& Satoh, K. An improved sodium dodecyl sulfatepolyacrylamide gel electrophoresis system for the analysis of membrane protein complexes. Electrophoresis 22, 1004-1007 (2001).

58. Porra, R. J., Thompson, W. A. \& Kriedemann, P. E. Determination of accurate extinction coefficients and simultaneous equations for assaying chlorophylls $a$ and $b$ extracted with four different solvents: verification of the concentration of chlorophyll standards by atomic absorption spectroscopy. Biochim. Biophys. Acta Bioenerg. 975, 384-394 (1989).

59. Li, Y., Scales, N., Blankenship, R. E., Willows, R. D. \& Chen, M. Extinction coefficient for red-shifted chlorophylls: chlorophyll $d$ and chlorophyll $f$. Biochim. Biophys. Acta Bioenerg. 1817, 1292-1298 (2012).

60. Kashino, Y. \& Kudoh, S. Concerted response of xanthophyll-cycle pigments in a marine diatom, Chaetoceros gracilis, to shifts in light condition. Phycol. Res. 51, 168-172 (2003)

61. Ozawa, S.-I., Kosugi, M., Kashino, Y., Sugimura, T. \& Takahashi, Y. 5'monohydroxyphylloquinone is the dominant naphthoquinone of PSI in the green alga Chlamydomonas reinhardtii. Plant Cell Physiol. 53, 237-243 (2012).

62. Hamaguchi, T. et al. A new cryo-EM system for single particle analysis. J. Struct. Biol. 207, 40-48 (2019).

63. Maki-Yonekura, S. et al. Hexameric and pentameric complexes of the ExbBD energizer in the ton system. Elife 7, e35419 (2018).

64. Zhang, J. et al. JADAS: A customizable automated data acquisition system and its application to ice-embedded single particles. J. Struct. Biol. 165, 1-9 (2009).

65. Zheng, S. Q. et al. MotionCor2: Anisotropic correction of beam-induced motion for improved cryo-electron microscopy. Nat. Methods 14, 331-332 (2017).

66. Rohou, A. \& Grigorieff, N. CTFFIND4: fast and accurate defocus estimation from electron micrographs. J. Struct. Biol. 192, 216-221 (2015).

67. Webb, B. \& Sali, A. Comparative protein structure modeling using MODELLER. Curr. Protoc. Bioinformatics 54, 5.6.1-5.6.37 (2016).

68. Pettersen, E. F. et al. UCSF Chimera - a visualization system for exploratory research and analysis. J. Comput. Chem. 25, 1605-1612 (2004).

69. Kim, D. N. et al. Cryo_fit: democratization of flexible fitting for cryo-EM. J. Struct. Biol. 208, 1-6 (2019).

70. Afonine, P. V. et al. Real-space refinement in PHENIX for cryo-EM and crystallography. Acta Crystallogr. Sect. D Struct. Biol. 74, 531-544 (2018).

71. Emsley, P., Lohkamp, B., Scott, W. G. \& Cowtan, K. Features and development of Coot. Acta Crystallogr. Sect. D Biol. Crystallogr. 66, 486-501 (2010).

72. Nicholls, R. A., Tykac, M., Kovalevskiy, O. \& Murshudov, G. N. Current approaches for the fitting and refinement of atomic models into cryo-EM maps using CCP-EM. Acta Crystallogr. Sect. D Struct. Biol. D74, 492-505 (2018).

73. Pintilie, G. \& Chiu, W. Assessment of structural features in Cryo-EM density maps using SSE and side chain Z-scores. J. Struct. Biol. 204, 564-571 (2018).

74. Pintilie, G. et al. Measurement of atom resolvability in cryo-EM maps with Qscores. Nat. Methods 17, 328-334 (2020).

75. Schrödinger, L. The PyMOL Molecular Graphics System. Version 2.4.1. (2021)

76. Moriarty, N. W., Grosse-Kunstleve, R. W. \& Adams, P. D. Electronic ligand builder and optimization workbench (eLBOW): a tool for ligand coordinate and restraint generation. Acta Crystallogr. Sect. D Biol. Crystallogr. D65, 1074-1080 (2009).

77. Schulte, T., Hiller, R. G. \& Hofmann, E. X-ray structures of the peridininchlorophyll-protein reconstituted with different chlorophylls. FEBS Lett. 584, 973-978 (2010).

\section{Acknowledgements}

K.K. is grateful to Prof. Nobuo Kamiya (Osaka City University) for providing laboratory access. K.I. would like to thank Mr. Yuzo Watanabe (Proteomics Facility, Graduate School of Biostudies, Kyoto University) for his help with mass spectrometric analysis. We are grateful to Ms. Yoko Kunishima and Ms. Kyoko Ishino for their technical assistance, to Prof. Tatsuya Tomo (Faculty of Science Division I, Tokyo University of Science) for providing spectral data of Halomicronema hongdechloris. We thank James Allen, DPhil, and Jeremy Kamen, M.Sc. Biology, from the Edanz Group (https://en-author-services. edanzgroup.com/), and Dr. David Mcintosh for editing this manuscript. This work was supported by grants-in-aid from the Japan Society for the Promotion of Science (Grant $18 \mathrm{H} 05175$ to Y.K.; $16 \mathrm{H} 04757$ to K.Y.; $16 \mathrm{H} 06554$ to K.I.; and 20K06684 to S.I., 20K06515 to T.H.; and $20 \mathrm{H} 05109$ and $20 \mathrm{~K} 06528$ to K.K.), the RIKEN Pioneering Project, Dynamic Structural Biology (to T.H. and K.Y.), the Cyclic Innovation for Clinical Empowerment (CiCLE) from the Japan Agency for Medical Research and Development, AMED (to T.H. and K.Y.), Innovations for Light-Energy Conversion (I ${ }^{4} L E C$; to Y.K.), and JST-Mirai Program Grant Number JPMJMI20G5 (to K.Y.)

\section{Author contributions}

K.S.-I., N.I.-K., E.Y., and K.M. optimized purification conditions. K.S.-I. purified the PSI trimer used in cryo-EM experiments. K.S.-I., N.I.-K., S.I., K.I., and Y.K. performed biochemical analyses. T.H. and K.Y. collected and analyzed cryo-EM data. K.K. built and refined the structural model. T.H., K.K., K.S.-I., K.Y., and Y.K. wrote the manuscript with input from all other authors.

\section{Competing interests}

The authors declare no competing interests.

\section{Additional information}

Supplementary information The online version contains supplementary material available at https://doi.org/10.1038/s41467-021-22502-8.

Correspondence and requests for materials should be addressed to K.K., K.Y. or Y.K.

Peer review information Nature Communications thanks Zhenfeng Liu and other, anonymous, reviewers for their contributions to the peer review of this work. Peer review files are available.

\section{Reprints and permission information is available at http://www.nature.com/reprints}

Publisher's note Springer Nature remains neutral with regard to jurisdictional claims in published maps and institutional affiliations.

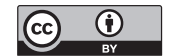

Open Access This article is licensed under a Creative Commons Attribution 4.0 International License, which permits use, sharing, adaptation, distribution and reproduction in any medium or format, as long as you give appropriate credit to the original author(s) and the source, provide a link to the Creative Commons license, and indicate if changes were made. The images or other third party material in this article are included in the article's Creative Commons license, unless indicated otherwise in a credit line to the material. If material is not included in the article's Creative Commons license and your intended use is not permitted by statutory regulation or exceeds the permitted use, you will need to obtain permission directly from the copyright holder. To view a copy of this license, visit http://creativecommons.org/ licenses/by/4.0/.

(C) The Author(s) 2021 